\title{
Peculiarities of using demonstrative pronoun ça in the modern French language
}

\section{[Les particularites de l'emploi du pronom demonstratif ça en français contemporain]}

\author{
Aleksei A. Zaitsev - Dinara G. Vasbieva
}

DOI: $10.18355 / X L .2020 .13 .01 .15$

\begin{abstract}
The paper discusses the use of the clipped form of the demonstrative pronoun ça in the modern French language, and the issue of language change mechanisms as well. The study is implemented on the fictional works by the French writers of the second half of the 20th century and early 21 st century. It also deals with decoding monological and dialogical genres of the modern French spoken language, performed within the framework of the project «The modern French language phonology». The study has revealed an extension of the scope of using the clipped form of demonstrative pronoun ça, that is displacing the synonyms cela and ce not only from direct, but also from indirect speech. Strengthening the neutralization process, when using demonstrative pronoun ça, used as a substitute for as cela and ce makes it possible to build up the language change mechanism chain.
\end{abstract}

Keywords: demonstrative pronoun ça, language change, colloquial speech, usage extension, modern French language

\section{Résumé}

L'article est consacré à l'analyse de l'emploi de la forme réduite du pronom démonstratif ça en français contemporain, ainsi qu'à la question des mécanismes des changements de langue. La recherche est mise en œuvre sur le matériel de la communication littéraire des écrivains français de la deuxième moitié du XXe-début $\mathrm{du}$ XXIe siècle, ainsi que sur celui des transcriptions des monologues et dialogues du français contemporain parlé, faites dans le cadre du projet «Phonologie de la langue française contemporaine». L'extension du domaine de l'emploi de la forme réduite du pronom démonstratif ça, qui déplace activement les synonymes de cela et ce non seulement au discours direct, mais aussi au discours indirect, est révélée. On y révèle l'extension du domaine de l'emploi de la forme réduite du pronom démonstratif ça, qui déplace activement les synonymes de cela et ce tenant non seulement discours direct, mais aussi du discours indirect. Le renforcement du processus de la neutralisation, lorsqu'on utilise le pronom démonstratif ça en tant que substitut de cela et ce, permet de construire une chaîne du mécanisme des changements de langue.

Mot clés: pronom démonstratif ça, changements de langue, discours familier, extension de l'emploi, français contemporain

\section{Introduction}

Il n'est pas besoin d'un long préambule pour justifier ce fait que le langage est un système complexe. Son système apparaît sans cesse en mouvement. Est-ce un mouvement illusoire, qui s'épuise sur place en efforts stériles? Ou bien le langage tend-il vers une fin idéale, dont il se rapprocherait à chaque étape de son évolution ? En effet, comme Budagov (1965) l'a souligné, «le langage se développe non seulement dans le passé, mais également dans le présent. Son mouvement se ressent 
même dans le synchronisme. Par conséquent, le concept du développement est tout aussi important pour le synchronisme que pour le diachronisme».

Nous connaissons l'histoire de certaines langues pendant des périodes de temps imposantes. Gnezdilova (2019) écrit que la langue est un fait historique, attesté sous des formes très variées, à des dates fort différentes, sur toute l'étendue de notre globe. Nous les voyons souvent se transformer avec une grande rapidité. Nous sommes donc en droit de nous demander quel est le sens de ces transformations, et à poser en d'autres termes la question des changements linguistiques.

Actuellement, le français traverse une période de transition. Cédera-t-il à la poussée néologisante, qui peut balayer les meilleurs acquis des transformations subies au cours de son histoire, se figera-t-il à tel point qu'il ne pourra subvenir à tous les besoins de communication, ou s'engagera-t-il dans la voie d'une raisonnable évolution?

Ces questions inquiètent non seulement les linguistes mais aussi tous les Français, soucieux de la vitalité et du prestige de leur langue.

Tout mouvement ne va pas sans heurts ni sans contradictions. Les débats déclenchés autour de l'avenir du français en sont un témoignage flagrant. Les esprits lucides se rendent parfaitement compte de la nécessité d'une réglementation de la langue, qui ne se produit pas toujours de façon efficace. Cette réglementation ne doit pas porter préjudice à son identité, car elle est censée suivre le rythme des changements qui s'opèrent dans le monde actuel. D'une part, il y a lieu de se récrier contre le flot d'innovations qui va à l'encontre du génie de la langue ; d'autre part, il est indispensable de créer constamment de nouvelles formes d'expression (lexicales ou grammaticales) pour être de son temps, au diapason de l'époque. De là, des opinions qui s'entrechoquent et qui, espérons-le, apporteront à la longue les meilleures solutions quant au choix des moyens d'expression. Comme le suggère Sauvageot: «La langue «commune» nouvelle doit être réglée de sorte que ceux qui s'en rendront maîtres puissent tout aussi bien goûter le classicisme français que ce qui a suivi. C'est faire appel à la continuité» (Sauvageot, 1978). Ce conseil, formulé il y a plus de 40 ans, ne perdra rien, et pour encore longtemps, de son actualité.

$\mathrm{Au}$ cours des dernières décennies a paru un nombre d'ouvrages visant justement à étudier les changements linguistiques au sein de la communauté parlante. Les linguistes s'intéressent à ce problème depuis très longtemps.

Il est à noter que les XXe et XXIe siècles sont marqués par les progrès considérables du français. Le développement rapide de l'économie, la diffusion de l'instruction, l'introduction d'internet dans les habitudes de communication y ont largement contribué (Galimova et al., 2019). Tout nouveau moyen de communication inventé bouleverse les habitudes et crée souvent de nouveaux comportements. Différents exemples sont à relever: l'imprimerie, le téléphone ... très peu de contemporains soupçonnaient ce qu'ils allaient permettre.

Nous nous proposons pour tâche d'analyser le système de la langue. Nous optons cependant pour une mise en lumière de l'étude de la parole, car seule l'analyse de la pratique peut fournir des preuves.

Notre recherche sur l'emploi du pronom démonstratif ça constitue une première tentative pour aborder la question du mécanisme des changements linguistiques, d'une manière objective et empirique. C'est que le système de toute langue vivante est instable. Il renferme en lui autant de causes de changement que le le système phonétique. Mais il y a une différence dans la manière dont s'effectue le changement des deux systèmes. Le changement grammatical ne porte jamais que sur les mots, et non sur les morphèmes en général.

La cause du changement grammatical est non dans les catégories de l'esprit, mais dans l'usage opéré par la langue. Le changement grammatical part toujours d'un

XLinguae, Volume 13 Issue 1, January 2020, ISSN 1337-8384, eISSN 2453-711X 
emploi donné et n'a par la suite chaque fois qu'une extension limitée. Ce n'est pas le système qui se modifie, comme dans certains changements phonétiques, mais seulement un des éléments du système ; et encore : dans un emploi donné.

\section{Revue de littérature}

En faisant une petite digression historique, il convient de noter que les quatre premières éditions du Dictionnaire de l'Académie française, exprimant les aspirations extrêmement puristes de leurs auteurs, avaient superbement ignoré le pronom ça. Mais en 1798, quelques années après qu'on ait beaucoup entendu chanter le refrain de la chanson «Ah ça ira, ça ira!», en pleine période révolutionnaire, le pronom ça s'est faufilé dans la cinquième édition du Dictionnaire avec la mention: «Ça se prend quelquefois pour cela, mais il est populaire et familier. Qu'est-ce que ça vaut? Donnez-moi ça». Dans la dernière édition, de 1835 , ça est déjà considéré comme une forme abrégée du pronom démonstratif cela : «Ça se dit par contraction, dans le langage familier, pour cela» (http://www.academie-francaise.fr/). Il n'est pas étonnant que, supplanté dans l'usage par ça, cela ait peu à peu été mis au ban des outils grammaticaux.

De nombreux chercheurs ont développé les bases théoriques de la mise en œuvre de la classification des pronoms français (Wolf, 1974; Gak, 2000; Serebrennikova, 1998; Blanche-Benveniste, 1984; et autres). Les travaux de ces chercheurs sont axés sur l'étude des caractéristiques de l'organisation et du fonctionnement du système des pronoms en tant que classe spéciale des unités linguistiques. Cependant, les formes distinctes des pronoms démonstratifs restent insuffisamment étudiées.

\section{Matériaux et Méthodes}

Le matériel de la recherche en question comprend des œuvres littéraires des écrivains français de la deuxième moitié du XXe - début du XXIe siècle (plus de 50 titres, pour un volume total de 10000 pages), ainsi que des transcriptions de monologues et dialogues du français contemporain parlé, effectuées dans le cadre du projet «Phonologie de la langue française contemporaine» (Phonologie du français contemporain - PFC).

Pour collecter des données empiriques, une analyse théorique de la littérature relative au sujet de recherche, linguistique, philosophique et psychologique, a été appliquée. Pour le traitement des données empiriques, il a été fait usage de méthodes de modélisation linguistique, ainsi que d'un traitement de données statistiques, l'interprétation de celles-ci étant effectuée par des méthodes de recherche comparative, transformationnelle et descriptive.

La recherche a été menée en trois étapes.

Au cours de la première étape, le choix de l'objet, du sujet et du problème de recherche, ainsi que l'analyse théorique, ont été effectués ; la base de recherche et la méthodologie ont été déterminées, l'hypothèse a été formulée.

Lors de la deuxième étape, on a révélé par la méthode de l'échantillonnage continu des phrases contenant la forme du pronom démonstratif ça. Les exemples donnés en guise d'illustration sont très loin d'épuiser leur nombre dans le corps du matériel étudié.

À la troisième étape, on a procédé à l'analyse et la synthèse des résultats de la recherche linguistique et proposé le modèle du changement linguistique.

\section{Résultats et discussion}

De nombreux chercheurs s'occupant de la syntaxe normative et stylistique de la langue française attirent l'attention sur l'expansion de l'emploi du pronom démonstratif ça, au détriment des pronoms cela et ce. Tous ces chercheurs partagent 
l'opinion que la forme ça, existant dans le langage familier, porte une certaine connotation sémantique de familiarité grossière, de négligence, de mépris, de tendresse, de compassion, etc.

Pour illustrer ce point de vue, on cite généralement un certain type de proposition, à savoir les propositions avec reprise ou anticipation, appelées propositions segmentées, dans lesquelles ça joue le rôle d'un moyen de mise en relief, en soulignant l'un ou l'autre des éléments de l'énoncé. La plupart des chercheurs se limitent à considérer des structures de ce type, réduisant ainsi le champ de leurs observations des exemples avec ça, ne désignant qu'un être vivant. Par exemple:

Et un homme, ça boit son café, le matin (Clavel, 1974); Les débutants, ça gaspille, ça boit beaucoup, et puis le moteur en a peut-être consommé une grande quantité (Frison-Roche R., 2001); Ça vous, amuse, vous ces petits cousins (Sagan, 2008).

On peut trouver la corrélation de ça avec le nom désignant une personne déjà dans les œuvres de $\mathrm{H}$. de Balzac. Par exemple: Elle [...] se croit si sainte; ça communie tous les mois (Balzac).

Correspondant aux noms désignant une personne, le pronom ça a une certaine valeur émotionnelle et expressive. Cependant, cette nuance estimée peut se perdre dans des constructions similaires, dès que ça est corrélatif d'un nom qui indique un objet ou un phénomène. Par exemple:

Je vous ai préparé deux guerbas: ça sent le bouc, mais c'est frais ... (FrisonRoche, 2001). Les pierres, c'est dur et ça ne bouge pas (Sartre, 2007).

Dans certains ouvrages consacrés à la syntaxe de la langue française, nous trouvons une indication sur l'emploi de la forme ça remplaçant le pronom démonstratif, cela ayant ses différentes significations dans le langage familier. Cependant, l'intérêt des chercheurs n'est pas focalisé sur cet aspect du problème, mais plutôt sur la capacité du pronom ça d'avoir une valeur expressive ou émotionnelle, dans certains cas très limités. Pourtant, la fréquence d'utilisation de la forme raccourcie ça augmente de plus en plus, principalement en raison de l'omission des formes neutres cela et ce. Une telle extension du fonctionnement du pronom démonstratif ça, son implication dans des constructions stylistiquement neutres, présente un intérêt particulier pour dégager le mécanisme des changements linguistiques.

Passons maintenant à l'analyse du matériel linguistique.

En tant que sujet, dans son sens général, ça peut être combiné avec des verbes de la sémantique la plus diverse. Par exemple:

1. C'est touchant que vos sentiments à l'égard du sénateur n'aient pas faibli avec le temps, mais vous pouvez m'étrangler si cela, ça ne changerait rien (Levy, 2016); Tiens, ça serait un joli titre pour un roman Un automne à San Francisco; Vu nos relations, ça m'étonnerait; Ça m'arrive (Levy, 2013).

2. C'est comme ça que j'ai été blessé d' un éclat d'obus à la hanche. Ça s'est justement passé sur le front de Voroneje ... (Téry, 1952); Et à quoi ça sert? (Rolland,1955); Ça n'est pas venu tout d'un coup (Vaillant-Couturier, 1987).

3. Tout ça ne dit pas par quelles voies, quand et où ficher ou foutre le camp a pris le sens de lever le camp en vitesse (Cohen, 1970); ... il faut «retourner les poches». Mais savez-vous ce que ça veut dire - juste les mêmes mots - à Poitiers ou à Niort? Ça veut dire; il faut «rapporter les sacs en papiers» (Cohen, 1970).

Comme on peut le constater, dans tous les exemples cités, ça paraît dans son sens général. Il n'est guère possible de voir dans ce type de constructions des nuances de la signification expressive ou émotionnelle. Les deux derniers exemples, tirés du texte de contenu scientifique, présentent un intérêt particulier puisqu'ils montrent la pénétration de la forme raccourcie ça dans des styles de la communication écrite, et la

XLinguae, Volume 13 Issue 1, January 2020, ISSN 1337-8384, eISSN 2453-711X 
perte progressive par cette forme de son rapport exclusif avec les styles populaire et familier.

Dans le même sens de généralisation, le pronom ça est largement utilisé par des auteurs qui font l'exposé à la première personne (parfois sous la forme d'un journal) : J.-P. Sartre, Vilar et al. Ainsi, dans le roman de J.-P. Sartre : "La Nausée", le pronom ça a complètement remplacé la forme cela. Par exemple:Pourquoi n'en aije pas parlé? Ça doit être par orgueil et puis, aussi, un peu par maladresse (Sartre, 2007).

L'emploi illimité de la forme raccourcie ça peut expliquer son envahissement progressif de la zone du pronom démonstratif ce dans la construction avec le verbe être (même dans les idiomes comme : «Ce n'est pas vrai »), et avec les verbes modaux : pouvoir, devoir. Par exemple:

1. Ça n'est pas juste (Frison-Roche R., 2001); Si je parle à propos de cette histoire, ça n'est pas que je mette en balance Flore (Courtade, 1954); Mais ça n'est rien, ça! (Téry, 1952).

2. La panne! Si près du but. Non. Ça n'était pas juste (Frison-Roche, 2001) ; Un verre de thé peut-être? Ça ne serait pas de refus (Téry, 1952).

3. Mais ça n'est pas un palais, c'est un métro pour aller son travail (Téry, 1952).

Dans le dernier exemple, on peut sentir l'envie de l'auteur du reportage de souligner l'importance du phénomène, ce qui entraîne l'emploi de la forme raccourcie ça au lieu de la forme traditionnelle atone ce - ce n'est pas...

Assez souvent, ça remplace le pronom démonstratif ce comme partie nominale du prédicat. Par exemple:Un drôle de gars ... un peu individualiste, c'est ça qui l'a perdu ; ... autrefois, à Londres, à Meknès, à Tokio ... j'ai eu des aventures. C'est ça qu'on m'enlève, à présent(Sartre, 2007).

Une caractéristique remarquable du français contemporain est que le pronom raccourci ça est en mesure de concurrencer, dans la fonction de sujet, les pronoms personnels de la 3ème personne. C'est le cas des constructions dans lesquelles le locuteur est indifférent à l'appartenance de genre du sujet, puisque le pluriel est perçu comme la désignation d'un ensemble indifférencié, ou plus souvent, lorsque la détermination quantitative est insignifiante pour les interlocuteurs.

L'emploi de ça en tant que sujet au lieu des pronoms personnels de la 3ème personne est lié d'une part à la structure de la phrase, et d'autre part au type du nom auquel correspond le pronom démonstratif. Souvent, dans ces cas, la forme ça est utilisée pour mettre en relief le sujet. Par exemple: - Et sinon euh, à Dijon vous avez toujours habité euh, au même endroit? - Euh, ben moi j'étais à Nlieu euh, ça fait un moment, ça fait, ça fait quatre cinq ans que je suis à Nlieu; Ouais, ça fait chaud parce que là, c'était l'équivalent d'un R.M.I., enfin pas quasiment quoi, c'est euh, pour la nounou; Ben ça dépend, non euh, on a, ça nous arrivait plusieurs fois d'héberger des gens euh, mais euh, comme ça quoi (https://public.projetpfc.net/hyperaudio/index.php?folder=21ama1\&type=g).

Le matériel de recherche montre qu'on peut parler d'une répartition particulière des fonctions entre des formes «concurrencées», un pronom démonstratif et personnel : pour le pronom ça, la norme du langage familier est la substitution d'un nom non-animé de n'importe quelle appartenance de genre, pour un pronom personnel - la substitution d'un nom désignant une personne.

Il existe une très large série de contextes dans lesquels ça est employé en tant que complément au discours direct et au discours indirect dans les ouvrages des écrivains de la deuxième moitié du XXe - début du XXIe siècle, ce qui reflète les particularités du français familier. Par exemple:1. Qui vous a fait ça? (Levy, 2016); Pourquoi aurait-il révélé ça au sénateur? (Levy, 2016);

2. Je vois ça! Que s'est-il passé? dit Lauren (Levy, 2005); 
3. En attendant, je vais imprimer ça et et le placer en évidence sur son bureau (Levy, 2015); Vous ne pouvez pas rater ça (Levy, 2013);

4. Daniel m'a lu de vos vers ... Ah, j'ai eu tort de vous raconter ça! (Gard, $2003)$; - Je ferai enlever ça - c'est une petite assiette portant le nom du locataire (Courtade, 1954) ; Si tu appelles ça aider! (Courtade, 1954); Tu fais le dégoûté ... j'ai connu ça, mon petit (Daix, 1994);

5. Pascal trouve ça absurde (Aragon, 2000); Le travail avec Marc, Jacques Roussel ne trouve pas ça très sérieux (Daix, 1994).

Le pronom ça en tant que complément d'objet direct est largement représenté dans le discours direct, mais ne s'est faufilé qu'une seule fois dans le texte de l'auteur - dans le rapport de Simona Teri. Par exemple:

Toute la presse marshallisée parle du "rideau de fer". Moi, j'appellerais plutôt ça le rideau de dollars ... ; On ouvre par son milieu la première poupée-boîte -...- et on trouve, à l'intérieur, une autre poupée pareille, mais de couleurs différentes... Quels cris de joie quand on déboîte tout ça (Téry, 1952).

En plus, le pronom démonstratif ça, dans la fonction à la fois de sujet et de complément d'objet, se rapporte aussi librement aux objets et aux phénomènes concrets mentionnés précédemment. Dans ce cas, ça est l'équivalent du pronom personnel de la 3 personne ou du nom correspondant.

1. Je suis sûr que tout va bien, il n'y a qu'à me secouer légèrement, ça cesse tout de suite (Aragon, 2000) ; Et. bien, on s'y attend, et on prend ça, il lui passe le flacon (Gard, 2003) ;

2. Il était considéré comme un lieu de rendez-vous comme un cataclysme, mais si ça devait frayer la voie à Pétain! (Aragon, 2000); Ténéré, ça devait se trouver derrière les grandes tours de grès? ... (Frison-Roche R., 2001);

3. Quand j'en fume une, c'est plutôt pour des raisons sentimentales, parce que ça vient de là-bas (Téry, 1952); Mon compagnon de voyage m'offre une cigarette russe et moi, je lui donne une gauloise en échange. Il n'en a jamais fumé, il trouve ça très fort, ça le fait tousser (Téry, 1952); Personne ne lui avait dit que parmi les vieilles à la forme des fenêtres aux colonnes, à la décoration, on pouvait reconnaître l'époque à la quelle ça avait été bâti ... (Aragon, 2000).

La corrélation du pronom ça avec des objets concrets est particulièrement et souvent exprimée dans les exemples du dernier type : dans les cas où il s'agit d'une grande quantité d'objets, ce qui découle clairement de la signification générale du pronom lui-même.

J'hésite: derière moi le boulevard conduit au coeur de la ville, aux grandes parures de feu des rues centrales ... Ça neme tente pas du tout ... (Sartre, 2007).

En français, le complément indirect exprimé par le substantif est toujours accompagné d'une préposition, qui est sa marque grammaticale. Donc, le complément indirect peut coïncider formellement avec certains types de compléments circonstanciels. Il arrive aussi que les deux termes soient voisins du point de vue sémantique. Cependant, les liens syntaxiques du complément d'objet et du complément circonstanciel avec le prédicat sont différents, ainsi que la nature du lien de la préposition avec le substantif. Le complément d'objet indirect peut aussi être exprimé par d'autres parties du discours, notamment par les pronoms (conjoints et indépendants): jelui parle, je pense à lui, et par les pronoms démonstratifs, y compris le pronom ça. Ça en fonction du complément d'objet indirect remplace de plus en plus la forme complète de cela, non seulement dans le discours direct, mais aussi dans le discours d'auteur. Par exemple:

1. J'ai eu des aventures, si cela peut te rassurer; et puis change de sujets, je n'ai pas envie de parler de ça(Levy, 2005) ; Non, pour ça rien n'est prévu (Levy, 2005); Laisse, dit Mia en la rejoignant, c'est à moi de m'occuper de ça, tu as assez de

XLinguae, Volume 13 Issue 1, January 2020, ISSN 1337-8384, eISSN 2453-711X 
travail en cuisine (Levy, 2013); Pas besoin de ça: à quoi sert-il de passer la soirée à se fiur soi-même si c'est pour être rattrapé en bout de course à son domicile?(Beigbeder, 1997);

2. C'est pour ça que vous n'aimez pas Whitman autant que lui (Gard, 2003); A quoi bon revenir sur tout ça? (Gard, 2003); Pendant que je pensais à tout ça, Claire a mis sa main sur mon épaule (Courtade, 1954);

3. Quoi! toutes ces angoisses, tout ce martyre pourça ... une petite pièce de rien du tout (Frison-Roche R., 2001) ;

4. Denise parlait froid de toutça (Aragon, 2000); Qui pensait de là-haut, du porche de Trocadéro où les Mercadier avaient fait halte, à quoi que ce fût au monde, à la foule, aux restaurants, aux bicoques, à la bouffée de musique berbère... Qui échappait de tout ça dans l'après-midi finissante (Aragon, 2000).

Tous les exemples ci-dessus montrent que la forme du pronom ça, remplaçant cela et ce, ne présente aucune valeur évaluative ou expressive. Cependant, son appartenance stylistique au langage familier ne permet pas de parler de la neutralisation finale de ce pronom.

Les exemples étudiés montrent que l'emploi du pronom indéfini tout avec le pronom ça peut renforcer la valeur générale de ce dernier. En même temps, l'expression «tout ça» correspond toujours à ce qui a déjà été dit, alors que le pronom ça peut lui-même correspondre à la partie de l'énoncé qui le suit. Par exemple:

On était gosse encore, ça nous faisait battre le coeur, les articles de Daudet, le chauffeur Baju... (Aragon, 2000); Ça ne t'ennuie pas trop de travailler comme ça, toujours seul? (Gard, 2003); Ça m'était presque égal, votre antipathie(Gard, 2003).

A vrai dire, dans de telles expressions, nous avons affaire à un moyen particulier de mettre en relief, de souligner les éléments d'expression. Ce type de phrase segmentée limite la capacité de ça à se rapporter à la partie du message qui le suit.

Par conséquent, le pronom démonstratif ça peut avoir une autre fonction dans la phrase française, il agit d'un moyen de mise en relief.

Il est à noter que l'une des plus grandes particularités des termes de proposition consiste en ce qu'ils peuvent fonctionner en position détachée. Dans cette position, ces termes se caractérisent par un ensemble de traits spécifiques qui les distinguent des termes correspondants non-détachés. Dans ce cas-là ils sont notamment beaucoup plus indépendants du point de vue syntaxique, sémantique et phonétique, et leur lien avec le terme régissant dont ils sont séparés par une pause (à laquelle correspond une virgule dans la réécriture) est relativement lâche. En conséquence, la proposition contenant un ou plusieurs termes détachés (segmentés) se caractérise par une intonation spécifique qui correspond à la segmentation de la proposition. Les termes en position détachée, en vertu de leur détachement syntaxique, sont liés non seulement au terme régissant, mais aussi à tout le noyau prédicatif (ou à la proposition en entier). Ceci est une conséquence inévitable de l'indépendance syntaxique et sémantique du terme détaché, ainsi que de la courbe mélodique particulière, qui affaiblissent son lien direct au terme régissant.

L'indépendance relative des termes détachés détermine leur mobilité positionnelle : tous les termes détachés sont susceptibles d'occuper n'importe quelle place dans la proposition, notamment de se trouver en position finale, initiale et médiane par rapport au sujet ou au prédicat.

Quant au pronom démonstratif ça, ce pronom est assez souvent employé en français moderne dans les phrases segmentées à côté du pronom personnel de la troisième personne. C'est surtout le cas de la reprise:

1. "Avedo», ça vient d'abies», disait-il (Vaillant-Couturier, 1987);

2. La ligne, ça vaut au moins cinq cents francs (Daix, 1994); 
3. Mais les fuites, ça peut compliquer les choses (Willard, 2001).

Contrairement à ce qui se produit avec une phrase segmentée par un pronom personnel à la troisième personne, qui est utilisé lors de la reprise pour mettre en relief le sujet ainsi que le complément d'objet direct et indirect, les constructions avec le pronom démonstratif ça ne sont utilisées que pour souligner le sujet.

Un autre type de phrase segmentée qui entraîne beaucoup plus largement le pronom démonstratif ça dans sa construction, c'est l'anticipation (l'un des types de la mise en relief, y compris la reprise).

1 Ça compte si peu, pour moi, en ce moment, mon départ, et ces quatre ans ... (Gard, 2003);

2 Ça met de l'animation, ce métro aérien (Daix, 1994);

Parmi les types de l'anticipation, il y a une construction dans laquelle ça anticipe l'infinitif prépositionnel qui suit le prédicat.

1. Mais ça te fatiguerait, Salomé, de poser ... (Vaillant-Couturier, 1987); Çavous amuserait de le voir de près? (Gard, 2003);

2. Ça l'amuse de voir un peu comment les débutants se débrouillent (Vaillant-Couturier, 1987);

3. Ça doit être joliment agréable de passer d'une vieille maison de bois à un appartement ultra-moderne! (Téry, 1952).

L'emploi de cette construction dans le discours direct et indirect est perçu comme une concurrence des tours présentatifs c'est ...qui, c'est ...que, placés en tête de la phrase avec les mots qu'ils encadrent.

Une forte tendance de l'expansion du pronom démonstratif ça se traduit par son emploi dans les phrases segmentées, dans la fonction du sujet. Dans ce cas, le prédicat a comme complément la subordonnée. A tire d'exemple citons :

1. ça l'aurait embêté que son secrétaire fût la tête (Aragon, 2000);

2. Ça l'agaçait que Pierrot se préoccupât du sort de Laure (Daix, 1994); Ça faisait bien longtemps qu'il n'avait pas été rencontré Mathieu (Daix, 1994).

Le dernier exemple peut être considéré comme une attaque de la structure avec ça contre le tour présentatif c'est. . . que (Ça faisait bien longtemps qu'il n'avait pas rencontré, Mathieu). Ce tour renforcé par le pronom ça est largement employé dans le registre familier. Ce phénomène est surtout reproduit par les écrivains dans le discours direct des personnages.

1. Ça, c'est irrésistible (Gard, 2003);

2. Ça, c'est la gare avec les quatre cafés (Sartre, 2007);

3. Dans un quart d'heure, Roberte a rendez-vous avec Marc et ça c'est sérieux! (Daix, 1994).

\section{Bilan}

La présente recherche concernant le pronom démonstratif ça en français contemporain montre l'extension du domaine de son emploi. Ce pronom démonstratif est employé aussi bien dans le discours direct que dans le discours indirect, ce qui peut être considéré comme un procédé stylistique particulier appliqué par de nombreux écrivains français - nos contemporains.

Le pronom ça a élargi ses emplois et s'introduit de plus en plus dans des contextes davantage variés qu'auparavant. Les résultats de notre étude permettent de constater que plus un mot est employé fréquemment au milieu de contextes différents, plus sa valeur risque d'en être modifiée. L'emploi fréquent recourt aux mots aussi bien dans leur sens que dans leur forme, et d'autant plus lorsqu'il s'agit de mots

XLinguae, Volume 13 Issue 1, January 2020, ISSN 1337-8384, eISSN 2453-711X 
expressifs. La valeur expressive du pronom démonstratif ça s'atténue rapidement à l'usage. Ce mot devient terne et fruste.

Il est toutefois impossible de parler de neutralisation complète du pronom démonstratif ça, utilisé comme substitut de cela et ce, puisque les structures avec ça appartiennent au style du langage familier et gardent la valeur de familiarité inhérente à ce dernier. En même temps, l'emploi fréquent des structures du langage familier et l'implication de ça dans le texte narratif de l'auteur, témoignent du changement en cours des caractéristiques stylistiques.

Ainsi, un bref aperçu du fonctionnement du pronom démonstratif ça montre qu'on peut en distinguer deux emplois : métaphorique et neutre, qui tendent à devenir homonymes. L'emploi de ça comme équivalent de n'importe quel nom animé porte un caractère occasionnel, ce qui conditionne un fort effet stylistique. A son second emploi (neutre, sans effet stylistique), le recours au pronom ça à la place de cela et ce, comme caractéristique du langage familier, s'efface de plus en plus au cours de cet emploi. L'extension de ce second ça dans le français d'aujourd'hui permet de dégager le mécanisme des changements de langue: extension de la combinaison $\rightarrow$ disparition d'un trait distinctif $\rightarrow$ changement sémantique $\rightarrow$ remplacement des relations normatives anciennes par de nouvelles formes.

Les changements de langue consistent en ce que la langue s'adapte le mieux aux besoins des sujets parlants. Mais si réel que soit ce procès, il n'est jamais définitif. La langue étant un instrument de communication ouvert à toutes les oscillations de l'esprit humain, seul un tri raisonnable couronné par l'usage décidera du sort des variantes d'expression.

\section{Bibliographic references}

ARAGON, L. 2000. Voyageurs de l'impériale. Paris: Gallimard. ISBN: 978-2-29611359-6.

BEIGBEDER, F. 1997. L'amour dure trois ans. Paris: Grasset and Fasquelle. ISBN: 9782246546511.

BLANCHE-BENVINISTE, C. 1984. Pronom et syntaxe. L'approche pronominale et son application au français. Pronoun and syntax. The pronominal approach and its application to French. Paris: SELAF. ISBN: 9782852972025.

BUDAGOV, R. A. 1965. Problems of language development. Moscow: Science. ISBN: 5-211-00391-8.

CLAVEL, B. 1974. La maison des autres. Paris: J'ai lu. ISBN 10: 2277125229 / ISBN 13: 9782277125228.

COHEN, M. 1970. Toujours des regards sur la langue française. Paris: Editions sociales. ISBN: 978-2-406- 05945-5.

COURTADE, P. 1954. Les circonstances. Nouvelles. Paris: La bibliothèque française. DAIX, P. 1994. Le dix-neuvième printemps. Moscou: Jupiter-Inter.

FRISON-ROCHE, R. 2001. Le rendez-vous d'Essendilène. Paris: J'ai lu. ISBN10: 2290316881 / ISBN-13: 978-2290316887.

GAK, V.G. 2000. Theoretical grammar of the French language. Moscow: Dobrosvet. ISBN 978-619-7408-19-5.

GALIMOVA, E.G. - KONYSHEVA, A.V. - KALUGINA, O.A. - SIZOVA, Z.M. 2019. Digital educational footprint as a way to evaluate the results of students' learning and cognitive activity in the process of teaching mathematics. Eurasia Journal of Mathematics, Science and Technology Education, vol. 15, n.8, em1732.ISSN: 1305-8223.

GARD, R. M. 2003. Les Thibaut. Paris: Editions Gallimard. 
GNEZDILOVA, E.Yu. - ZAITSEV, A.A. 2019. Metodika prepodavaniya istorii zarubezhnoy literatury v vuze: problemy i resheniya, Methods of teaching the history of foreign literature at the university: problems and solutions. In: Humanities and education, vol. 11, n. 1, pp. 43-49. ISSN: 2079-3499.

LEVY, M. 2005. Vous revoir. Paris: Robert Laffont. ISBN 10: 2221102789 / ISBN 13: 9782221102787.

LEVY, M. 2013. Elle et Lui. Paris: Versilio. ISBN: 9782221157831.

LEVY, M. 2016. Un sentiment plus fort que la peur. Paris: Versilio. ISBN: 2221127137.

ROLLAND, R. 1955. L'âme enchantée. Moscou: Editions en langues etrangères.

SAGAN, F. 2008. Château en Suède. Paris: Julliard. ISBN-10: 2260017665; ISBN13: 978-2260017660.

SARTRE, J-P. 2007. La Nausée. Paris: Gallimard. ISBN: 9782070368051.

SAUVAGEOT, A. 1978. Français d'hier ou Français de demain, French of yesterday or French of tomorrow. Paris: Collection language question.

SEREBRENNIKOVA, E.F. 1998. Ways of presenting the person personal pronouns in French. Irkutsk: Publishing House of Irkutsk University. Available online: http://cheloveknauka.com/sposoby-predstavleniya-litsa-lichnymi-mestoimeniyami-vofrantsuzskom-yazyke

TERY, S. 1952. Une française en Union Soviétique. Paris: Editions sociales.

VAILLANT-COUTURIER, P. 1987. Enfance. Paris: Messidor. ISBN: 2-909422-453 .

WILLARD, M. 2001. Des yeux qui voient. Paris: Les éditeurs français réunis.

WOLF, E.M. 1974. Grammar and semantics of pronouns. Moscow: Science.

Words: 5394

Characters: 33994 (18,88 standard pages)

assoc. prof. Aleksei An. Zaitsev, PhD

Foreign Languages Department

Russian State Agrarian University - Moscow Timiryazev Agricultural Academy

Timiryazevskaya Str., 49

127550 Moscow

Russia

a.zaizev@mail.ru

assoc. prof. Dinara G. Vasbieva, PhD

Foreign Languages Department

Financial University under the Government of the Russian Federation

Leningradsky prospect 49

125993 Moscow

Russia

dinara-va@list.ru

XLinguae, Volume 13 Issue 1, January 2020, ISSN 1337-8384, eISSN 2453-711X 